\section{A MODEL AND TREATMENT FOR AUTISM AT THE CONVERGENCE OF CHINESE MEDICINE AND WESTERN SCIENCE: FIRST 130 CASES}

\author{
L. Silva, M. Schalock, R. Ayres \\ Teaching Research Institute, Western Oregon \\ University, Monmouth, OR, USA
}

Objective: To present a model for early intervention in autism and demonstrate the efficacy of a treatment based on Chinese medicine on measures of autism. In Chinese medical terms, the model explains autism as a partial closure of the sensory orifices due to toxicity or block. Translated into Western medical terms, an impairment of sensory regulation underlies the abnormal behaviors and developmental delays seen in autism. Treatment is a qigong massage methodology.

Methods: Children with autism under six years of age were assigned to treatment or wait-list conditions. 130 children were treated and the results compared with 45 wait-list controls. The treatment is a five-month qigong massage protocol that was implemented by trained parents via trained support staff. The effects of treatment on the main symptoms, autistic behavior, social/language delay, sensory and self-regulatory impairment, as well as on parenting stress, were observed and compared.

Results: Treatment had a large effect size $(p<$ .0001) on measures of sensory and self-regulation. Evaluations done by pre-school teachers demonstrated improvement in measures of autism - small effect size $(p<.003)$, and were confirmed by evaluations done by parents - large effect size $(p<$ $.0001)$. There was a large decrease $(p<.0001)$ in parenting stress.

Conclusion: Sensory impairment is an important factor in the development and severity of autism. Treatment of young children with autism with a qigong massage methodology results in a decrease in sensory and self-regulatory impairment and a reduction in severity of measures of autism.

\section{CSF-LACTATE AS A MARKER OF MITOCHONDRIAL DISORDERS EVEN IN CHILDREN AFTER BRIEF SEIZURES}

M. Magner ${ }^{1}$, K. Szentiványi ${ }^{1}$, I. Švandová ${ }^{2}$, P. Ješina ${ }^{1}$, M. Tesařová ${ }^{1}$, T. Honzík ${ }^{1}, J^{\prime}$. Zeman ${ }^{1}$

${ }^{1}$ Department of Children and Adolescent Medicine, First Medical Faculty, Charles University, ${ }^{2}$ Department of Physiology, Faculty of Science, Charles University, Prague, Czech Republic

Background: An increased level of lactate in body fluids serves as a biochemical marker in assessing patients with inherited metabolic disease; especially in children with mitochondrial disorders that arise from isolated or combined defects of respiratory chain complexes and ATP-synthase. On the other hand increased lactate may be falsely considered to be the result of seizures in children with mitochondrial disorders. Therefore we ascertained the diagnostic value of lactate and alanine in blood and cerebrospinal fluid (CSF) in children with mitochondrial disorders $(n=24)$, epilepsy $(n=32)$ and psychomotor retardation $(n=23)$.

Results: Average blood and CSF lactate levels were significantly higher in children with mitochondrial disorders $(3.87 \pm 0.48$ and $4.43 \pm 0.55 \mathrm{mmol} / \mathrm{l})$ than in children with epilepsy $(1.72 \pm 0.13$ and $1.62 \pm 0.04$ $\mathrm{mmol} / \mathrm{l})$ or psychomotor retardation $(1.79 \pm 1.40$ and $1.68 \pm 0.06 \mathrm{mmol} / \mathrm{l})$. Blood and CSF alanine levels were also significantly higher in children with mitochondrial disorders than in children with epilepsy or psychomotor retardation. The CSF lactate levels of children with epilepsy were similar whether the samples were obtained $3 \pm 0.6$ hours after an attack of brief seizures or from children without history of recent seizures.

Conclusion: Even in children who have recently suffered short-lasting seizures, elevated cerebrospinal fluid lactate level is a reliable marker pointing to the mitochondrial origin of disease. Brief seizures lasting less than 2 minutes do not increase lactate concentration in the CSF. Repeated lactate analyses are sometimes necessary, because lactate may be elevated only intermittently in children with mitochondrial disorders.

Work was supported by grants IGA MZ NS 105613/2009 and IGA NS 9782-4/2008 